\title{
ACTITUDES PROPOSICIONALES Y CONOCIMIENTO SENSIBLE EN TOMÁS DE AQUINO
}

\author{
Jörg Alejandro Tellkamp \\ Pontificia Universidad Javeriana. Bogotá (Colombia)
}

\section{RESUMEN}

Según el criterio tradicional, la epistemología de Tomás de Aquino explica el conocimiento diferenciado e intencional de la realidad en términos de una doctrina del intelecto. En oposición a esta tendencia, se puede constatar la existencia de otro tipo de conocimiento complejo que se realiza a través de la actividad de los sentidos. Un análisis de la teoría tomasiana de los sensibles revelará una posición sorprendentemente cercana a la teoría moderna de la psicología del sentido común, es decir, una teoría que describe la percepción y la conducta de los animales y los seres humanos en términos de la experiencia cotidiana de objetos singulares.

Palabras clave: Tomás de Aquino, Conocimiento intencional, Psicología del Sentido Común.

\section{ABSTRACT}

Propositional attitudes and sensible knowledge in Thomas Aquinas. The traditional approach to Thomas Aquinas' theory of knowledge suggests that complex, intentional knowledge is only available to the intellect. Against this, there is strong evidence for an alternative view, which holds that the perceptual grasp of the sensible world renders a complex, however, different kind of knowledge. An analysis of Aquinas' theory of the sensibles reveals a stance which shows a striking resemblance to modern Folk Psychology, i.e., a strong commitment to describe animal and human perception and behavior in terms of daily life experience of singular objects.

Key words: Thomas de Aquino, Intentional knowledge, Folk Psychology.

\section{INTRODUCCIÓN}

Normalmente la experiencia cotidiana de los objetos materiales no se presenta como problema y mucho menos como problema filosófico. En la mayoría de los casos estamos acostumbrados a asumir que nuestro aparato sensorial nos lleva a un conocimiento correcto de lo que es el caso. Esto es válido incluso admitiendo que los sentidos suelen engañarnos bajo determinadas circuns-

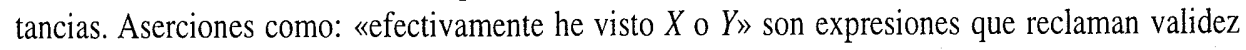
cognitiva y que presuponen que el mundo es tal como nos aparece. Este concepto de adquisición y justificación de conocimiento perceptivo se apoya filosóficamente en lo que se ha llamado una 
Psicología del Sentido Común. Tradicionalmente este punto de vista implica un realismo con respecto al conocimiento; con respecto a la percepción se ha etiquetado esta perspectiva con el epíteto de realismo ingenuo 0 «naiv». ${ }^{1}$ Todo parece indicar que éste es el punto de vista de Tomás de Aquino en el contexto de la discusión de contenidos cognitivos, siendo éstos los resultados de procesos perceptivos. Lo que percibimos por medio de nuestros sentidos es fiel copia del mundo sensible, siempre y cuando el aparato sensorial funcione adecuadamente y cuando las circunstancias, p.ej., la distancia entre sujeto y objeto, lo permiten.

Hay que advertir que este artículo se centrará únicamente en el análisis de lo que son contenidos perceptivos, sin abordar el tema de la veracidad de los mismos. Dado el caso que Tomás asume — ceteris paribus - la verdad de lo que percibimos, no hay necesidad de enfocar el tema desde el punto de vista del escéptico.

¿Qué es un contenido perceptivo para Tomás? Es, in nuce, aquel tipo de conocimiento particular que se da bajo determinaciones materiales y que es adquirido por medio de los sentidos exteriores e interiores. Tomás denomina estos contenidos sensibilia .0 sea, sensibles y de éstos hay que resaltar dos aspectos: 1) los sensibles representan contenidos cognitivos complejos, sobre todo en su manifestación de sensibile per accidens; 2) su ocurrencia no depende - por lo menos directamente - de la racionalidad del ser ni de intencionalidad consciente alguna. Se verá que este punto no sólo es de especial interés para la explicación de la percepción de animales, sino también para la de los seres humanos.

Como ya fue insinuado, la discusión de los sensibles parte en Tomás, tal como en otros autores del siglo XIII, de un tipo de Psicología del Sentido Común. Tendencias actuales de esta corriente filosófica suelen poner el énfasis en el análisis de las actitudes proposicionales, es decir, de aquellos estados mentales que muestran cierta direccionalidad o intencionalidad. ${ }^{2}$ Actitudes proposicionales son, según el concepto de Russell, explicitadas de la forma «que $X$ es el caso». ${ }^{3}$ Me parece que existe en este aspecto una semejanza entre las teorías de autores históricamente tan divergentes como, p.ej., Jerry Fodor y Tomás de Aquino, lo que se evidencia en la importancia que ambos le dan al funcionamiento cotidiano de nuestro aparato cognitivo. Una breve descripción de los puntos esenciales de la Psicología del Sentido Común servirá como punto de referencia para lo que sigue:

1) Las explicaciones del sentido común funcionan únicamente con base en una teoría de actitudes proposicionales. Éstas tienen, según Fodor, las siguientes características: i) pueden ser evaluadas semánticamente, lo cual es importante para determinar-la veracidad de contenidos perceptivos; ii) tienen un poder causal, sobre todo con miras al comportamiento

1. En la tradición filosófica hay que resaltar dos textos que marcaron el punto de vista escéptico: Sextus Empíricus y su crítica del realismo ingenuo através del tercer tropo (Pyrrhoneae Hypotyposes I, 91-98) y, naturalmente, George Berkeley en su Principles of Human Knowledge. Philosophical Works Including the Works on Vision, M.R. Ayers (ed.), Londres, Everyman, 1993.

2 En lo que sigue haré referencia a J. Fodor, Psychosemantics. The Problem of Meaning in the Philosophy of Mind, Cambridge (Mass.)/Londres, MIT Press, 1987, pp. 2-16.

3 Cf. B. Russell, An Inquiry into Meaning and Truth, Londres, Routledge, 1992, p. 21. Véase también R. Chisholm, Perceiving: A Philosophical Study, Ithaca/Londres, Cornell University Press, 1957, cap. 10. 
animal y humano; iii) las generalizaciones de la Psicología del Sentido Común pueden ser aplicadas a las actitudes proposicionales.

2) Los enunciados sobre estados mentales y sobre la percepción hacen referencia a procesos mentales que están dotados de un poder causal. Esto garantiza el éxito predictivo y explicativo de esta teoría.

3) Las teorías del sentido común son científicamente respetables. La teoría de los sensibilia forma parte de una Psicología del Sentido Común y, por lo tanto, se puede explicar una variedad de fenómenos con ella.

4) Esta teoría es, en la práctica cotidiana de explicar y predecir, imprescindible. No sabríamos cómo referirnos a estados intencionales como deseos y percepciones, sin hacer alusión a actitudes proposicionales.

\section{TOMÁS DE AQUINO Y LOS SENSIBLES}

Visto superficialmente, la percepción parece no desempeñar un papel importante en la filosofía de Tomás de Aquino. Comparando la mera cantidad de sus escritos sobre este tema con, p.ej., las reflexiones de Alberto Magno sobre el particular, resulta evidente que estamos tocando una teoría periférica. El factor cuantitativo, sin embargo, no es decisivo para establecer la calidad de una teoría; y si la atención se torna sobre la peculiar importancia de la percepción como fundamentum cognitionis, cambia drásticamente la perspectiva. Tomás afirma que es a través de nuestros sentidos que adquirimos las similitudes sensoriales (phantasmata o especies sensibles) necesarias para la abstracción intelectiva, a través de la cual se llega al conocimiento de los rasgos universales de las cosas. Conocimiento de cualquier tipo es, en su concepto, inconcebible sin un acto perceptivo previo. Estas pocas observaciones señalan que la percepción tiene una relevancia para el conocimiento en general, razón por la cual no es asombroso que Tomás reflexione sobre las causas, condiciones y los resultados cognitivos de los actos perceptivos.

La teoría de los sensibles (sensibilia) es un instrumento para determinar estos resultados cognitivos. A través de ellos, la tradición aristotélica pretende aclarar la complejidad del conocimiento sensible. El análisis de los sensibles parte de una descripción de los datos rudimentarios de la captación sensible de la realidad, es decir de las cualidades y de las cantidades sensibles, o sea, aquello que Locke denomina primary y secondary qualities, y lo que Tomás llama sensibles comunes y sensibles propios, respectivamente.

\section{EL SENSIBILE PER SE}

Tomás establece en la descripción del orden sistemático de la percepción las condiciones necesarias para cualquier acto perceptivo. Siguiendo al Averroes Latinus y a Alberto Magno, denomina

4 La literatura sobre este tema es muy escasa y bastante vieja. Se encuentran observaciones introductorias en R. Brennan, Thomistic Psychology: A Philosophic Analysis of the Nature of Man, Nueva York, Macmillan, 1941, pp. 120-124. 
a estos elementos sensibilia per se. Antes de profundizar este aspecto, cabe señalar que Tomás no utiliza el término sensibile de manera unívoca, pues puede significar el objeto material y sensible mismo, o puede referirse a las propiedades percibidas, que están per se o per accidens en los sentidos. ${ }^{5}$ Debo aclarar que me referiré en lo que sigue a la segunda acepción de este concepto.

En el contexto de la especificación de contenidos perceptivos, Tomás diferencia conceptualmente entre lo que es percibido por sí mismo (per se) y lo que es percibido accidentalmente (per accidens). ${ }^{6}$ Los sensibles accidentales serán objeto de discusión más adelante. Los sensibles per se se dividen, según la tradición aristotélica, en sensibles propios y sensibles comunes. Un texto tomasiano nos introduce a la terminología:

«Sic autem se habet ad cognoscendum res, inquantum similitudo [v.g., sensibile: J.A.T.] rerum est in sensu. Uno modo, primo et per se; sicut in visu est similitudo colorum et aliorum propriorum sensibilium. Alio modo, per se, sed non primo; sicut in visu est similitudo figurae vel magnitudinis, et aliorum communium sensibilium. Tertio modo, nec primo nec per se, sed per accidens; sicut in visu est similitudo hominis, non inquantum est homo, sed inquantum huic colorato accidit esse hominem». ${ }^{7}$

La primera determinación — primo et per se - se aplica a los sensibles propios, y la segunda - per se, sed non primo-, a los sensibles comunes. Lamentablemente Tomás no es muy específico con respecto a la diferencia semántica de lo que es per se y de lo que es primo. Los dos términos parecen señalar una función constitutiva de aquellos sensibles de los que se predica per se y/o primo. A pesar de la semejanza semántica de ambos conceptos, solamente la propiedad de poder ser recibido primo se relaciona con los sensibles propios, mientras que de ambos, sensibles propios y comunes, se predica el per se. La locución de algo que es percibido per se aclara que es esencial o necesario para la obtención un contenido perceptivo. En el Averroes Latinus y en Alberto Magno se habla de contenidos perceptivos que son captados essentialiter, y esto corresponde a la locución de per se en Tomás. ${ }^{8}$ El paralelismo semántico entre lo que ès esencial y lo que es per se indica que

Además: J. de Tonquédec, Les principes de la philosophie thomiste. La critique de la connaissance, París, Gabriel

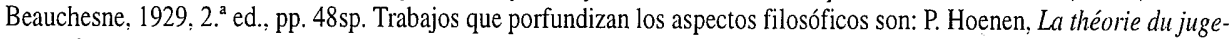
ment d'après P. Thomas d'Aquin, Roma, Universidad Gregoriana, 1953, 2. ${ }^{a}$ ed., pp. 252sp. Además, A. Hayen. L'intentionnel dans la philosophie de Saint Thomas, París/Bruselas, Desclée de Brouwer, 1954; 2. ${ }^{2}$ ed., pp. 11, 129 y 207.

5 Así, p.ej., en Quaestio Disputata De Anima a. 13 c., donde Tomás menciona explícitamente las species sensibilium. En el contexto de este texo tomasiano, sensibile no puede significar otra cosa que el objeto material mismo.

6 El sensible es el quod de lo que percibimos y no el quo, término que cae bajo consideraciones causales.

7 S.th. I q. 17 a. 2 c. Aquí, el término similitudo es aparentemente análogo al de sensibile. Cf., sin embargo, con respecto a Aristóteles, R. Sorabji, «Intentionality and Physiological Processes: Aristotle's Theory of Sense-Perception», en Martha C. Nussbaum, y Amélie Oksenberg Rorty (eds.), Essays on Aristotle's De Anima, Oxford, Oxford University Press, 1992, pp. 195-225. Además, T. Ebert, «Aristotle on What Is Done in Perceiving», Zeitschrift für Philosophische Forschung, 37 (1983), pp. 181-198.

8 Cf., p.ej., Averroes, De Anima II, 64 p. 226, $19-22$ (ed. Stuart Crawford): «Hec [v.g. comprehensio sensuum: J.A.T.] igitur est intentio eius quod est accidentaliter, quod est oppositum ei quod est essentialiter. Ista enim sunt comprehensibilia sensuum secundum quod sunt sensus, non secundum quod sunt aliqui sensus». Magnus Albertus, Opera Omnia, volumen 35, Secunda Pars Summae de Creaturis q. 34 a. 2, August Borgnet (ed.). París 1896, p. 103a, 18-20; «[...] Color in oculo non recipitur sine quantitate et his quae sunt quantitati annexa essentialiter». $C f$. líneas 25 ss.: «Aliud autem est 
los sensibles per se tienen que ser aquellos rasgos de lo percibido sin los cuales ningún acto cognitivo de percepción sería posible. Es decir, que únicamente podemos hablar de percepción cuando, implícita o explícitamente, nos referimos a las propiedades cualitativas y cuantitativas de objetos sensibles. De esta manera se puede constatar que los sensibles per se representan los elementos necesarios de toda percepción. En contraste con lo que es per se, el término primo parece indicar una conexión causal que rige entre el objeto sensible y la ocurrencia de actos perceptivos. Esto se explicará a continuación.

\subsection{El sensible propio}

Al determinar aquello que se percibe de manera primordial, Tomás recurre al vocabulario de la Summa de Creaturis de Alberto Magno. ${ }^{9}$ En aquel texto, los sensibles propios son caracterizados por dos aspectos peculiares: por un lado, por el hecho de ser los elementos necesarios dentro del complejo perceptivo. Por otro lado, en cambio, se entienden estos sensibles como el resultado directo de una causa que ejerce su eficacia sobre el aparato sensorial; es por eso que su occurrencia tiene un estatus primordial, acuérdese del primo, en cuanto sea el primer eslabón en la cadena que causa contenidos perceptivos.

En este contexto, la expresión primo se tiene que atribuir a la justificación ontológica de alteraciones físicas. Cabe resaltar que la teoría de los sensibles propios incluye una física de alteraciones o inmutaciones: «Nam sensibilia propria primo et per se immutant [cursivas mías: J.A.T.] sensuum» (S.th. I q. 78 a. 3 ad 2). Desde un punto de vista de causalidad eficiente es importante la pregunta: ¿cómo puede un objeto, a través de sus propiedades cualitativas, alterar físicamente un cuerpo, es decir, un órgano sensorial?

Un indicio clave es la mención que Tomás hace de la cualidades de la tercera especie: «[...] Non omnia accidentia habent vim immutativam secundum se; sed solae qualitates tertiae speciei, secundum quas contingit alteratio. Et ideo solae huiusmodi qualitates sunt obiecta sensuum [...]» (S.th. I q. 78 a. 3 ad 1). Un cambio (alteratio) sustancial o accidental de un objeto se produce solamente cuando la alteración es durable. ${ }^{10}$ De esta manera hablamos de un cambio accidental cuando, p.ej., un objeto blanco llega a ser negro.

sensatum [sc. sensibile commune], quod ideo per se senitiri dicitur, quia sua intentio in sensu imprimitur coniuncta sensibili proprio; et hoc est proximum subiectum sensatae speciei, quod est magnitudo, un qua ut in subiecto proprio est omnis qualitas sensibilis». También, o.c., p. 104, 9s.: «[...] Sensatum proprium est, quod agit primo in sensum[...]».

9 Cf. S.th. I q. 78 a. 3 ad 2. Véase al respecto Alberto o.c., q. 34 a. 2, pp. 298a ss. (ed. Borgnet).

10 In De Gen. et Corr. I, c. 4 1. 10 p. 300 a (ed. Leonina): «[...] Quando id quod transmutatur est passio permanentis, est alteratio [...]». Tomás parece además relacionar etimológicamente «alteratio» con «alterum», con lo cual se indica la diferencia temporal de cualidades en un objeto ante y post alterationem. Cf. In Phys. V, 4 n. 679 (ed. Marietti): «Huic enim generi alludit hoc commune nomen, quod est alteratio: nam alterum solet dici quod differt secundum qualitaten. Loquimur autem nunc de qualitate, non secundum quod quale invenitur in genere substantiae, secundum quod differentia substantialis dicitur praedicari in eo quod quale: sed quali passivo, quod continetur in tertia species qualitatis, secundum quod quale dicitur aliquid pati aut non pati, ut calidum et frigidum, album et nigrum, et huiusmodi». Véase también In De Gen. I c. III, 8 p. 293 (ed. Leonina): «[...] Nulla forma substantialis est per se sensu perceptibilis, sed solum intellectum, cuius obiectum est quod quid est [...], formae autem quae sunt sensu per se perceptibilis, sunt qualitates tertiae speciei, quae ob id dicuntur passibiles, quia sensibus ingerunt passiones, ut dicitur in Praedicamentis». 
En el contexto de la alteración de los sentidos, sólo se toman en consideración aquellas cualidades que pueden realmente alterar ( $p a t i$ ) los órganos. Esta cualidad conlleva el nombre de qualitas passibile, expresión que Tomás emplea en su exposición sobre las cualidades en Summa Theologica I-II q. 49 a. 2. El accidente «cualidad» es subdivido en cuatro categorías (species), de las cuales solamente una es perceptible. La lista abarca i) hábito, ii) estados potenciales, ${ }^{11}$ iii) pasión y iv) figura. La tercera y cuarta especie de la cualidad representan estados cualitativos actuales. La tercera parece constituir la estructura de fondo de un objeto, lo que está in profundum, mientras que la cuarta forma describe la superficie del objeto. Solamente la tercera es capaz de ser captada por los sentidos, siendo la propiedad intrínseca in profundum de una característica perceptible. Las cualidades pasibles circunscriben a su vez los objetos formales de cada una de las cinco potencias perceptivas, siendo los objetos formales lo visible (colores), lo audible (sonidos), etc. ${ }^{12}$ Como objeto formal no se entiende un determinado objeto material, sino una característica común a todo aquello capaz de captar justamente esa característica. El objeto formal es el definiens de una potencia cognitiva. ${ }^{13}$ Así, p.ej., la potencia visual es definida por su habilidad de ver colores y no por determinados objetos colorados.

El recurso a la doctrina de las cualidades es necesario para esclarecer la locución de «primo» en el contexto de los sensibles propios. Los sensibles propios son primordialmente captados, ya que se supone que aquello que es el efecto mayor en una cadena de causalidades es ontológicamente superior. La dependencia causal de los demás sensibles con respecto a los sensibles propios se traduce adicionalmente a una dependencia lógica. Este aspecto, sin embargo, no podrá ser desrrollado aquí, para no sobrepasar el enfoque delimitado de este artículo.

\subsection{Los sensibilia communia}

Al igual que John Locke, Tomás de Aquino supone que las propiedades cuantitativas de un objeto son esenciales para el proceso de percepción. ${ }^{14} \mathrm{~A}$ diferencia de las primary qualities del filósofo inglés, las cantidades sensibles desempeñan en el pensamiento del Aquinate un papel subordinado. La captación de estos sensibles es, como ya fue indicado, esencial para la percepción, sin embargo, se basa sobre la inmutación causada por los sensibles propios. Por esta dependencia causal, los sensibles comunes no son percibidos primo. ${ }^{15}$ No obstante, son esenciales para cualquier acto de percepción, ya que no se puede concebir un contenido perceptivo que no contenga de alguna manera una referencia a aspectos cuantitativos de lo percibido. La expresión «sensible común»

11 S.th. I-II q. 49 a. 2 c.: «[...] Naturalium autem qualitatum quaedam sunt secundum id quod aliquid est in potentia $[\ldots] »$.

12 In DA II, 13 p. 118, 20s (ed. Leonina): «[...] Dicuntur sensibilia illa [v.g. propria] que propria sunt singulis sensibus $[\ldots] \gg$.

13 S.th. I q. 1 a. 3 c.: «Est enim unitas potentiae et habitus consideranda secundum obiectum, non quidem materialiter, sed secundum rationem formalem obiecti: puta homo, asinus et lapis conveniunt in una formali ratione colorati, quod est obiectum visus». Cf. también A. Kenny, Action, Emotion and Will, Londres/Nueva York, Routledge \& Kegan Paul, 1963, p. 189.

14 S.th. I q. 78 a. 3 ad 2: «Sensibilia vero communia omnia reducuntur ad quantitatem».

15 Ibídem: «Quantitas autem est proximum subiectum qualitatis alterativae, ut superficies coloris. Et ideo sensibilia communia non movent sensum primo et per se, sed ratione sensibilia qualitatis [...]». 
(sensibile commune) deriva de la suposición de que algunas propiedades sensibles de objetos pueden ser percibidas por más de una potencia sensitiva. Es éste otro punto en que difieren de los sensibles propios, los cuales pueden ser percibidos por una y sólo una potencia sensitiva. ${ }^{16}$ Por definición, no existe un y sólo un sentido correspondiente a un sensible común.

Tomás determina, siguiéndo a Aristóteles y a sus comentaristas, la existencia de cinco sensibles comunes: movimiento (motus), reposo (quies), número (numerus), figura (figura) y extensión (magnitudo). ${ }^{17}$ Estos cinco términos no tienen, al contrario de lo que parecen, un mismo valor epistemológico. Los dos términos «número» y «extensión» forman la base para todos los demás sensibles comunes. Aparentemente este modelo de la dependencia de algunos sensibles comunes de otros más fundamentales tiene su orígen en Alberto Magno. ${ }^{18}$ Solamente la extensión y el número pueden ser denominados en un sentido estricto como cantidades. Los otros tres sensibles comunes, reposo, movimiento y figura, se derivan de éstos.

La figura de una cosa no es una cantidad «pura», ya que es, tal como lo expresa Tomás de manera enigmática, una cualidad de la cantidad. ${ }^{19}$ Se puede hablar de figuras en objetos sensibles solamente presuponiendo que ese objeto tenga una extensión tridimensional: «[...] Consistat ratio figurae in terminatione magnitudinis» (S.th. I q. 78 a. 3 ad 2). También «movimiento» y «reposo» dependen de la extensión de un objeto sensible. Solamente en el caso de tener un concepto cuantificable de una cosa material se puede predicar «movimiento» y «reposo» de ella. «Reposo» a su vez, depende conceptualmente de «movimiento», porque únicamente es pensable como ausencia de movimiento. ${ }^{20}$

De los sensibles comunes es sobre todo el del «número» el que crea dudas acerca de la coherencia de la teoría de los sensibles comunes como tal. Es notoria la desidia con la que Tomás trata al sensible común numeris. Probablemente, la falta de atención se debe a que él mismo se pudo haber dado cuenta de la deficiencia teórica de este sensible. ¿Cuáles son los problemas relacionados con éste?

Es parte de la definición de un sensible común el hecho de que sea percibido esencialmente (per se), lo que implica una captación inmediata de este sensible. El elemento definitorio, en cambio, no compagina con la observación cotidiana de que las propiedades numéricas de objetos no son percibidos de esta manera, es decir, inmediatamente. Podríamos decir que un adulto puede ver, sin contarlas conscientemente, cinco naranjas, pero hay que constatar que se llega rápidamente a los límites de semejante captación instantánea de números. Veinte o mil naranjas no pueden ser vistas distintamente sin emplear ciertos métodos de contarlas y sin tener un concepto de lo que es un número. Tomando en consideración esta reflexión, podría dudarse si la captación de mil naranjas

16 In DA II, 13 p. 118, 22ss. (ed. Leonina): «[...] Alio modo dicuntur sensibilia illa que communiter senciuntur ab omnibus sensibus».

17 Cf. In DA II, 13 p. 119, 46ss (ed. Leonina).

18 Cf. Albertus Magnus: De anima, p. 103b ss. (ed. Stroick).

19 S.th. I q. 78 a. 3 ad 2: «Figura autem est qualitas circa quantitatem [...]».

20 In DA II, 25 pp. 175, 183-185 (ed. Leonina): «[...] Manifestum est etiam quod quies comprehenditur ex motu, sicut et tenebra per lucem: est enim quies priuatio motus $[\ldots]$ ». 
constituye un acto de percepción de un sensible común. ${ }^{21}$ Evidentemente, esta inquietud no es respondida por el Aquinate, al igual que la pregunta de si los niños o los animales superiores pueden percibir los detalles numéricos del campo sensible. También se tiene que tomar en cuenta la crítica de George Berkeley: solamente se puede hablar coherentemente de números, haciendo referencia a un sistema de números y no a los objetos mismos que son contados. Los números son un producto de la mente y por lo tanto existen exclusivamente en ella. ${ }^{22}$

Los problemas hasta aquí enunciados abren el camino a un interrogante más global: si el sensible común numerus no es plenamente compatible con la exigencia de una percepción per se, es decir, esencial e inmediata, entonces ¿no cabría la duda de si la descripción de todos los demás sensibles comunes excede los límites de lo meramente físico e inmediato? ¿No sería entonces más prudente, tal como lo propone Berkeley, suponer estructuras mentales preestablecidas? Se verá más adelante que lo suscitado en esta pregunta es una característica de los sensibles accidentales.

Efectivamente, Tomás concede en el comentario al De Anima que, según un modo de decir, los sensibles comunes son percibidos accidentalmente. ${ }^{23}$ Es evidente que aquí se trata de una mera forma de hablar en cuanto los sensibles comunes estén subordinados a los sensibles propios y no en cuanto tengan algo en común con los sensibles accidentales. Tomás aclara la distinción entre los sensibles accidentales y los demás sensibles diciendo: «sensibilia communia non senciuntur per accidens ab aliquo sensuum, set per se a pluribus». ${ }^{24}$ Los sensibles comunes no pueden ser percibidos per accidens, porque son el resultado directo de una alteración orgánica. Esta aseveración es sorprendente, ya que Tomás había demostrado que solamente los sensibles propios son la consecuencia de una inmutación física, que se da, a su vez, por la causalidad de las cualidades de la tercera especie.

La percepción a nivel de los sensibles esenciales presupone la capacidad de distinguir cualidades y cantidades sensibles. El color negro es distinguido del blanco, al igual que los objetos grandes de los pequeños. Es crítica la pregunta de cómo se tienen que entender los verbos entender 0 conocer en este contexto. Es cierto que Tomás mismo utiliża en el contexto de la percepción casi

21 Es interesante anotar que esta crítica fue anticipada por Nemesio de Emesa. Según él, los sentidos tienen un acceso muy limitado a las propiedades numéricas representadas en objetos sensibles. Él define este límite en un número de tres o cuatro. Tomando la ayuda del criterio de inclusión (así se puede entender el término «immissio», que determina la cantidad de elementos que pueden ser incluidos en una experiencia perceptiva, anota que toda referencia perceptiva que excede el número de tres o cuatro tiene que ser respaldada por la memoria o la razón (mens). Cf. Nemesio de Emesa, De Natura Hominis, traduction de Burgundio de Pise, G. Verbeke, y J.R. Moncho (eds.), Leiden, E.J. Brill, 1975 (= Corpus Latinum in Aristotelem Graecorum Suppl. 1), cap. 6, p. 77, 69-76: «Igitur magnitudinem quidem dupliciter comprehendit, quandoque quidem solus visus, quandoque vero cum memoria et mente; numerum autem eorum quae videntur, qui super tria vel quattuor secundum unam immissionem videtur, et motum et figuram et multos angulos habentia nunquam solus, sed semper cum memoria et mente, Non enim potest quinque et sex et septem et plura sine memoria coadunare; ita neque sex angulos habentia et octo et multos angulos habentes figuras».

22 Cf. G. Berkeley, o.c., I, 12 p. 93: «Number is so visibly relative, and dependent on man's understanding, that it is strange to think how anyone should give it an absolute existence without the mind. We say one book, one page, one line; all these are equally units, though some contain several or the others. And in each instance it is plain, the unit relates to some particular combination of ideas arbitrarily put together by the mind».

23 Cf. In DA II, 13 p. 119, 75-79 (ed. Leonina).

24 In DA II, 25 p. 175, 157s. (ed. Leonina). 
exclusivamente el verbo latín iudicare, insinuando así una actividad relacionada con las capacidades intelectivas mediante la cual tipos iguales de datos son diferenciados. Siguiendo el punto de vista intelectualista, iudicare debería ser traducido por «juzgar». Existen, sin embargo, razones de peso contra tal interpretación.

En Summa Theologica I q. 78 a. 4 ad 2 se parte del supuesto de que cada sensus proprius es definido por su objeto específico u objeto formal. Los sentidos exteriores tienen la capacidad de distinguir diferentes realizaciones del objeto formal. 1) Por un lado, puede un sentido distinguir diferentes realizaciones de un objeto formal, como cuando el ojo distingue el negro del blanco, siendo ambos colores realizaciones del objeto formal «color». 2) Por otro lado, se distinguen mediante los sentidos propiedades que pertenecen a diferentes objetos formales, como cuando se distingue el blanco de lo amargo. Esta segunda forma de distinguir diferentes objetos formales pertenece propiamente a la actividad del sentido común (sensus communis).

El hecho de que Tomás utilice el verbo iudicare para referirse a la distincción entre realizaciones diversas de un objeto formal y la distinción entre varios objetos formales, puede llevar a una confusión respecto al mencionado verbo. ${ }^{25}$ Esta confusión consiste en atribuir a los sentidos una capacidad quasi-intelectiva mediante la cual consciente y conceptualmente se diferencian los objetos formales descritos. Esta interpretación intelectualista no puede, sin embargo, dar razón de la diferencia semántica que existe entre juzgar y distinguit: Ya que ambos verbos son alternativas viables para una traducción del verbo latín iudicare, trataré de argumentar en favor del verbo distinguir. ${ }^{26}$ Un criterio en contra del empleo de la traducción «juzgar» radica en que éste implica el uso de las facultades racionales. Tomás, sin embargo, es enfático al determinar que los actos de percepción no están relacionados con acto racional alguno. ${ }^{27}$ Adicionalmente, se pueden aducir los siguientes puntos en favor del verbo distinguir.:28

1) Distinguir es un verbo de éxito (achievement verb), mientras que un juicio errado sigue siendo un juicio.

2) Algo puede ser distinguido de manera clara u opaca, pero no de manera verdadera o falsa. Para los juicios, en cambio, los criterios de verdad son esenciales.

3) Juzgar es un verbo proposicional, distinguir es cognitivo. Esto significa que distinguir no depende de proposiciones previamente reconocidas como verdaderas, como es el caso en los juicios, los cuales llegan a una conclusión mediante un proceso discursivo de una pro-

$25 C f$. el siguiente artículo, sobre el cual basaré parte del argumento T. Ebert, «Aristotle on What Is Done in Perceiving», Zeitschrift für Philosophische Forschung, 37 (1983), pp. 181-198. Ebert demuestra con base en un análisis lingüístico del término griego krinein que al nivel primario de la percepción no se puede hablar tanto de actos de juicios, sino más bien de aquellos de distinción.

26 A pesar de que en la Edad Media se aplicaba el verbo discernere en contextos filosóficos, no es casi empleado en las discusiones sobre la percepción que opera en un margen aristotélico. El verbo de uso es iudicare. Tal vez esta utilzación un tanto limitada se deba a que traductores como Moerbeke no fueron conscientes de la gama semántica del griego krinein, empleando solamente el verbo latino semánticamente más estrecho iudicare. Como ejemplo, véase S.th. I q. 78 a. 4 ad 2: «[...] Sensus proprius iudicat de sensibili proprio, discernendo ipsum ab aliis quae cadunt sub eodem sensu, sicut discernendo albo a nigro vel a viridi».

27 Cf. Hoenen, o.c. p. 252: «On voit que le 'iudicium sensus' n'a qu' une analogie lointaine avec le jugement veritable [i.e., le jugement intellectuel: J.A.T.]».

28 Cf. Ebert, o.c., p. 184. 
posición a otra. Distinguir está directamente relacionado con el objeto del cual algo se distingue; juzgar se refiere a la verdad de una proposición.

4) Distinguir designa una actividad temporalmente limitada, no así juzgar.

5) Distinguir puede ser laborioso o no, juzgar, en cambio, no lo es.

6) La mayoría de las características que son aplicables al verbo distinguir también son aplicables a verbos como oír, ver, etc. El elemento común de todos estos verbos es un objeto cognitivo directo. Esto también es válido para verbos que presuponen los anteriormente mencionados, tales como «observar» o «escuchar». «Distinguir» implica, por ende, la actualización de una potencia cognitiva.

7) En favor del verbo distinguir como indicativo de la acción propia de los sentidos también hay que aducir que incluso los animales no racionales perciben el mundo de manera diferenciada. La interpretación intelectualista excluiría a todo ser viviente, exceptuando al ser humano, de una percepción coherente. El sensus proprius de una oveja es básicamente capaz de cumplir las mismas funciones que los sentidos de los seres humanos, a pesar de que tenemos que constatar que las ovejas no tienen capacidad de expresión verbal. ${ }^{29}$ Las diferencias entre animales perfectos y seres humanos se dan solamente con respecto a la reacción y la captación de situaciones complejas, las que Tomás también denomina intentiones.

De todas estas consideraciones resulta que las potencias sensitivas no juzgan, en el sentido de reflexión proposicional, sobre su objeto formal, sino que sencillamente distinguen diferentes episodios de un mismo tipo de objeto.

\subsection{Conocimiento a través de la percepción: los sensibilia per accidens}

Ver solamente un color o los rasgos de una cosa u oír un sonido, no constituye en sí un conocimiento de la cosa que se percibe. Si bien se tiene que destacar el papel causal de los sensibles per $s e$, Tomás constata que tienen un contenido cognitivo o intencional que pasa desapercibido al nivel de la descripción causal. Para acercarse a dicho contenido, es imprescindible transcender este nivel y dar razón de un tipo de conocimiento perceptivo diferenciado. ${ }^{30}$ Un determinado objeto blanco no es reconocido como «Sócrates» en virtud de su blancura, ya que no da razón del contenido proposicional representado en este objeto, es decir «que $X$ es el caso y que $X$ es Sócrates». El reconocimiento de que Sócrates es la cosa blanca percibida, se da como consecuencia de la captación previa de haber percibido un objeto blanco, es decir, un sensibile per se. ${ }^{31}$ Sin embargo, éstos no son suficientes, si bien necesarios, para poder captar el contenido proposicional. En cuanto se refiere a

29 Cf. S.th. I q. 78 a. 4 c.: «Considerandum est autem quod, quantum ad formas sensibiles, non est differentia inter hominem et alia animalia: similiter enim immutantur a sensibilibus exterioribus».

30 Esto se hace evidente en constataciones como la siguiente: S.th. I q. 78 a. 4 c.: «Necessarium est ergo animali quod percipiat huiusmodi intentiones, quas non percipit sensus exterior»

31 In DA II, 13 p. 119, 56-62 (ed. Leonina): «[...] Secundum accidens sensibile dicitur ut si dicamus quod Diarris uel Sortes est sensibile per accidens, quia accidit ei esse album: hoc enim sentitur per accidens quod accidit ei quod sentitur per se; accidit autem albo, quod est sensibile per se quod sit Diarris; unde Diarris est sensibile per accidens». 
la necesidad de los sensibles per se, Tomás constata que Sócrates como Sócrates no es perceptible, sino solamente como objeto blanco. ${ }^{32}$

Tomás afirma que la percepción accidental de Sócrates, es decir, un ser humano individual, o de una naranja, es algo que se da en el ámbito de la percepción. Teniendo en cuenta que la cognición perceptiva de un objeto se da a causa de la captación de proposiciones, y no a causa de una alteración física de los órganos, cabe la pregunta: ¿por qué se amplía de tal manera el concepto de lo que es un contenido percepivo a lo que ya no está relacionado directamente con el proceso físico de alteración orgánica?

Para formular una respuesta, hay que empezar por distinguir los significados que tiene el concepto de sensibile per accidens. ${ }^{33}$ Los diferentes significados de este concepto se basan en dos suposiciones: 1) un sensible accidental tiene que ser un accidente de un sensibile per se;2) tiene que ser percibido por un sujeto. De esto deduce Tomás los diferentes significados, que no son radicalmente diferentes ni incompatibles, pero que ayudan a formular un criterio para diferenciar los modos de referencia perceptiva.

1) La expresión sensibile per accidens significa en primera instancia la conexión elemental de experiencias sensoriales. Con base en la actividad de los sentidos propios se puede predicar algo sobre los objetos de otro sentido. Éste es el caso cuando «vemos» que el limón es ácido. En el ejemplo, la vista percibe accidentalmente lo que es percibido por el gusto per se. ${ }^{34}$ Parece no ser necesario que el sujeto esté en posesión de los conceptos de aquellas cosas que se perciben accidentalmente de esta manera.

2) El segundo significado dado por Tomás trasciende al ámbito de la experiencia sensorial inmediata, partiendo de la afirmación de que los sentidos son incapaces de captar los rasgos universales (aliquid uniuersale) de las cosas y que esto solamente se puede lograr por el intelecto. Como es sabido, este proceso es denominado abstracción. El intelecto agente llega a formular la universalidad de los phantasmata, los cuales denuda de sus condiciones individualizantes. Tomás se esfuerza en demostrar que el intelecto no es la única facultad cognitiva que puede acceder a universales. Lo interesante es que también por la vía de los sentidos se pueden captar algunos rasgos universales de objetos perceptibles. A diferencia del intelecto, que en su manifestación de ratiocinatio alcanza discursivamente lo universal, las facultades sensitivas lo captan inmediatamente (statim) a través de los sensibles accidentales, a los cuales los rasgos universales están unidos analíticamente. Esto se da, por ejemplo, en casos en que alguien ve a una persona hablar y percibe inmediatamente que esta persona vive. De esta manera parece que se puede percibir mediante los sentidos una característica universal de un ente particular, es decir la vida de un ser humano. ${ }^{35}$

32 In DA II, 13 p. 119, 59ss. (ed. Leonina): «[...] Hoc enim sentitur per accidens quod accidit ei quod sentitur per se; accidit autem albo, quod est sensibile per se, quod sit Diarris; unde Diarris est sensibilis per accidens. Vnde nichil patitur ab hoc in quantum huiusmodi».

33 Véanse las explicaciones en In DA II, 13 p. 120s. (ed. Leonina), sobre todo p. 120, 162ss. «[...] Restat uidendum qua ratione dicatur aliquid sensibile per accidens».

34 Ibídem, líneas 175-181. Cf. también In De Sens. 17 p. 94, 119s. (ed. Leonina).

35 In DA II, 13 p. 121, 184-190 (ed. Leonina): «Non tamen omne quod intellectu apprehendi potest in re sensata, potest dici sensibili per accidens, set quod statim ad occursum rei sensate apprehenditur intellectu, sicut statim cum uideo aliquem loquentem uel mouere se ipsum, apprehendo per intellectum uitam eius, unde possum dicere quod uideo eum uiuere». 
3) La tercera significación de sensibile per accidens es la más interesante desde un punto de vista filosófico. Su significado es básicamente el de la captación de una proposición o intentio individual bajo un aspecto universal, siempre basándose sobre la percepción de cualidades y cantidades sensibles. De esta manera la percepción del color «blanco» y de una determinada figura lleva, junto con el conocimiento previo de la forma universal «humanidad», a la percepción de «este ser humano»: «[...] Cum uideo coloratum, percipio hunc hominem uel hoc animal $[\ldots] » .36$

Es notable cierta similitud con la segunda acepción de sensibile per accidens, en la medida en que en ambas se describe la percepción de algo universal. La diferencia entre las dos acepciones reside, sin embargo, en el modo de relacionar lo particular con lo universal. En el ejemplo de la segunda acepción se vio que la unión conceptual de «movimiento autónomo» (movere se ipsum) y «vida» es necesaria. La tercera acepción, en cambio, no puede hacer alusión a tal vínculo necesario. La asignación de un predicado universal con base en las cualidades percibidas se puede dar solamente en conjunto con la realización de otra potencia cognitiva, es decir, con la vis aestimativa o la vis cogitativa. Para poder percibir distintamente que en determinado lugar está un ser humano particular, se requiere de un conocimiento previamente adquirido de lo que es un ser humano. Sin esta prenoción no se podría razonablemente decir que lo que está en el campo visual es un ser humano. En la segunda acepción no se requiere que el sujeto tenga una prenoción de «vida», cuando ve a un ser humano y «ve» que éste vive. El movimiento propio de los seres presupone necesariamente que éste se realice únicamente en seres vivientes, sin importar que el sujeto esté consciente de este hecho o no. Lo blanco, invocado en el ejemplo de la tercera acepción, se puede realizar en una multiplicidad de objetos, así que se hace necesario indicar si el objeto blanco es Sócrates o un hombre de nieve.

Los sensibles accidentales se distinguen fundamentalmente de los sensibles esenciales, lo cual ya lo indica la terminología empleada. La diferencia entre ambos consiste principalmente en la característica de los sensibles esenciales de ser resultado de un proceso físico en la periferia orgánica, mientras que los sensibles accidentales tienen que ser entendidos en términos de la captación de algo que es el caso, es decir, de la intentiones. Los sensibles accidentales constituyen un conocimiento sensorial complejo de lo que es el caso. Los sensibles esenciales, a su vez, representan los elementos a través de los cuales los sensibles accidentales adquieren su nitidez cognitiva, ya que cada episodio perceptivo necesariamente tiene que hacer alusión a las cualidades y las cantidades inherentes al objeto sensible. Sin embargo, el conocimiento sensorial, intencional, de una proposición no puede ser alcanzado únicamente al nivel de los sensibilia per se. Los sensibles accidentales no son percibidos directamente, es decir, como resultado inmediato de una alteración de la periferia orgánica, sino solamente de una manera derivada. ${ }^{37}$ Para utilizar el vocabulario de Thomas Reid o Gilbert Ryle, los sensibles accidentales son expresiones de perception y no de sensation. ${ }^{38}$

36 In DA II, 13 p. 121, 192s. (ed. Leonina).

37 De Tonquédec, o.c., p. 50.

38 Cf. Th. Reid, Essays on the Intellectual Powers of Man, ed. A.D. Woozley, Londres, 1941, cap. 16. G. Ryle, The Concept of Mind, Harmondsworth, Penguin, 1990, cap. VII. 
Los sensibles accidentales no son resultado de una reflexión consciente y racional. Sin embargo, ¿no significa su contenido proposicional una incursión en lo que es genuinamente intelectivo? A esta pregunta se puede formular la siguiente respuesta: existen básicamente dos tipos de acceso cognitivo al mundo y, por lo tanto, existen dos tipos de contenidos cognitivos, uno intelectivo y otro sensitivo. Desde luego, el conocimiento universal es adquirido por el intelecto; sin embargo, parece existir una forma de conocimiento complejo independiente de estructuras intelectivas. Contra una semejanza entre sensibles accidentales y los contenidos cognitivos del intelecto se puede argumentar que las capacidades racionales no son vistas como condición necesaria para la adquisición del conocimiento sensible, ya que es evidente que los animales tienen el último, pero no el primero.

Esto no significa, sin embargo, que las capacidades racionales no puedan desempeñar el papel de condición suficiente de conocimiento sensible en los seres humanos. Los contenidos cognitivos basados en la percepción, es decir, en los sensibles accidentales, son de tipo proposicional. Siempre que un sujeto se refiere perceptivamente a un objeto sensible, obtiene un contenido cognitivo «que $X$ es el caso». Esta especificación tiene como consecuencia una reevaluación del hiato tradicionalmente sostenido entre percepción superior humana y percepción inferior animal, cuestión que indirectamente es tematizada en el comentario al De Anima II, 29, texto en el cual Tomás evalúa la relación entre el intelecto y las capacidades sensitivas.

Lo interesante de la discusión representada en este texto es una aserción que indica que la capacidad de captar proposiciones no tiene como requisito necesario habilidades lingüísticas y, por ende, racionales. Explícitamente determina que - al menos - los animales superiores están en condiciones de captar objetos bajo aspectos no directamente perceptibles. ${ }^{39} \mathrm{La}$ experiencia visual de una oveja al ver un lobo no representa primordialmente un objeto oscuro de determinada forma. Instintivamente huye, no propter indecentiam coloris, sino, porque percibe aquel objeto como un enemigo de su naturaleza (quasi inimicum naturae). ${ }^{40}$ La captación instintiva de la intentio del lobo no implica que la oveja lo perciba bajo un aspecto universal (sub natura communi), sino bajo el aspecto práctico de ser término o principio de una pasión. Los animales superiores perciben intenciones solamente en cuanto éstas estén vinculadas con una acción de apetencia o de fuga. La conexión de los datos perceptivos per se con las intentiones son producto del instinto o de la vis aestimativa. De esta manera y de acuerdo con el ejemplo anteriormente citado, una oveja no reconoce a su madre como «esta oveja» bajo el aspecto universal de la «ovejidad», sino solamente en cuanto la madre sea fuente de alimento (in quantum [agnum:J.A.T.] est ab ea lactabiblis). ${ }^{41}$

La formación del conocimiento perceptivo en animales superiores y en seres humanos es, en el fondo, idéntica: ambos llegan, por medio de los sentidos, a un conocimiento sensorial captando un aspecto no perceptible, es decir, una intentio, la cual, a su vez, remite a un contexto práctico (en animales) o cognitivo universal (en seres humanos). A pesar de la habilidad de los seres humanos

39 Los así llamados animales inferiores se excluyen de esta determinación, ya que tienen solamente una phantasia indeterminata de un objeto perceptivo. Por lo tanto, no perciben suficientes propiedades sensibles. Cf. In DA II, 29 p. 194 (ed. Leonina).

40 S.th. I q. 78 a. 4 c.

41 In DA II, 13 p. 122, 211 ss. (ed. Leonina). 
de percibir la intentio de una cosa sub natura communi, se pueden aducir razones implícitas para no conectar el conocimiento perceptivo humano con las habilidades lingüísticas y racionales. Se puede argumentar, desde un punto de vista del sentido común, que no estaríamos en condiciones de razonablemente interpretar el comportamiento humano si partiéramos de la suposición de que únicamente la posesión de conceptos lingüísticamente expresables condujesen a un conocimiento perceptivo coherente. Una gran parte del poder explicativo de una teoría del sentido común se echaría a perder si limitásemos la teoría de la percepción al hecho de tener capacidades lingüísticas. Adicionalmente, parece que las creencias que especifican al objeto de la percepción no son necesarias para poder coherentemente decir que un animal o ser humano esté percibiendo..$^{42}$ Por lo tanto, las percepciones no son opiniones ni convicciones en el sentido de una conceptualización consciente. Sin embargo, esto no implica que el sujeto percipiente no pueda estar convencido de lo que percibe. Los contenidos perceptivos tienen un poder causal y, por esta razón, pueden producir una opinión o creencia sobre el objeto sensible. Lo inverso, no obstante, no parece ser el caso, ya que no necesitamos convicciones conscientes para poder percibir. ${ }^{43}$

Otro argumento contra un vínculo necesario entre el lenguage y la adquisición de conocimiento perceptivo radica en el hecho de que podemos explicar el último sin tener que recurrir a capacidades lingüísticas. Parece ser el caso que los contenidos perceptivos, típicamente representados en los sensibilia per accidens, no se basan solamente en los sensibles esenciales, sino también en una estructura de conocimiento previamente adquirido. En la terminología propuesta por John Searle, se habla de un conocimiento de fondo (background-knowledge). ${ }^{44}$ Este fondo cognitivo representa un conocimiento latente o disposicional que se actualiza siempre cuando determinados factores externos se den. Estos factores determinan la direccionalidad de los estados intencionales, es decir, estados como la percepción. Por ejemplo, la visión de un objeto siempre está acompañada de un conocimiento disposicional sobre el objeto visual, es decir, que es indispensable saber lo que se está percibiendo. No tiene sentido expresar la frase «yo veo un taxi» si no sé lo que son taxis. En analogía a esto podemos apreciar el ejemplo de là oveja y el lobo. La oveja no puede referirse perceptivamente al lobo como lobo si no sabe que allá está un animal que potencialmente puede acabar con su existencia. Obviamente, el fondo cognitivo de las ovejas es más limitado que el de los seres humanos; sin embargo, el principio que rige este punto es básicamente el mismo. Lo importante de esta digresión radica en que las disposiciones cognitivas de fondo no tienen que ser actualizadas de manera consciente, o sea. Conceptual, sino que es plenamente suficiente que se den, siempre y cuando un factor externo, un estímulo, las actualice.

42 T. Crane, «The Non-Conceptual Content of Experience», en T. Crane (ed.), The Contents of Experience: Essays on Perception, Cambridge, Cambridge University Press, 1992, pp. 136-157. Aquí, pp. 141 y 149. Cf. para la conexión entre lenguaje y percepción D.W. Hamlyn, «Perception, Sensation and Non-Conceptual Content», The Philosophical Quarterly; 44 (1994), pp. 139-153.

43 Esto es, según Tomás, el caso, ya que tener una opinión (opinio) está ligada con una creencia firme (fides), la cual no poseen los animales. No obstante, sería absurdo negarle a los animales una referencia perceptiva coherente. $C f$. In DA II, 29 p. 194, 158ss. (ed. Leonina).

44 Cf. J. Searle, Intentionality: An Essay in the Philosophy of Mind, Cambridge, Cambridge University Press, 1983, pp. 37-78 y $141-159$. 
La teoría de un conocimiento de fondo disposicional para explicar un conocimiento perceptivo complejo, i.e., intencional, abre el camino a la posibilidad de poder asumir estructuras cognitivas racionales latentes al igual que no racionales, sin correr el peligro de sucumbir a los riesgos que implica la suposición de una reflexión consciente y conceptual sobre lo que percibimos. La presencia del objeto perceptible y el proceso perceptivo son dos sucesos simultáneos, o, más bien, idénticos, lo que hace improbable un raciocinio conceptual previo. La suposición de un discurso racional y consciente al nivel de la percepción crearía además la tendencia de socavar la igualdad sensitiva de animal y ser humano, hecho que es enfáticamente defendido por Tomás. ${ }^{45}$

Aparte de estas razones implícitas contra una íntima conexión entre lo sensitivo e intelectivo, podemos aducir razones explícitas que se encuentran en el corpus tomasiano. ${ }^{46}$ En resumida cuentas, saber algo intelectivamente no tiene el mismo significado que percibir. ${ }^{47}$ Tomás hace además alusión a la noción aristotélica que diferentes potencias cognitivas se diferencian de acuerdo con sus objetos y funciones. Podemos encontrar en Tomás los siguientes argumentos en contra de la mencionada conexión esencial entre lo sensitivo y lo intelectivo:

1) Los sensibles accidentales se refieren a un objeto particular determinable a través de una intentio universal. Los contenidos intelectivos, en cambio, se caracterizan per definitionem por su referencia a un universal, obtenido por vía de la abstracción de la condiciones individualizantes de un phantasma.

2) Los sensibles accidentales son los productos de un acceso inmediato al mundo sensible, lo cual es una propiedad que comparten con los sensibles esenciales. La inmediatez de los actos perceptivos tiene que ver con que la percepción se realiza generalmente de manera inconsciente, sin reflexión, sin necesitar de otros actos cognitivos como en el caso del intelecto agente. En el momento de ver un taxi, no se percibe inmediatamente un objeto amarillo (sensibile proprium) y de tales dimensiones (sensibile commune), sino que es visto inmediatamente como taxi, habiendo captado la proposición «que allá está objeto $X$ con las características $a, b, c$ y que $X$ es un taxi».

3) Los actos perceptivos se diferencian de procesos intelectivos en que aquéllos no juzgan, sino que distinguen. También aquí se ve la carencia de cualquier reflexión racional en la percepción.

Estos tres puntos solamente demuestran que la percepción no tiene una conexión intrínseca con lo intelectivo; sin embargo, no demuestran que carezcan de relación alguna. Como se ha visto, cada ser tiene su fondo cognitivo específico, que contiene un conocimiento general sobre las características esenciales de läs cosas percibidas. En los seres humanos este conocimiento previo es racional, en los animales, meramente instintivo. En los seres humanos este conocimiento es un factor concomitante o disposicional de nuestra percepción, que es activado automáticamente cuando un determinado estímulo llega a los órganos. Es por eso que bajo condiciones normales reconocemos visualmente los taxis, sin expresamente reflexionar sobre la clase de objeto que tenemos fren-

45. Cf. In DA II, 28 p. 190, 190-198 (ed. Leonina).

46 Remito otra vez a In DA II, 29 (ed. Leonina).

47 Cf. In DA II, 28 p. 187, 9-11 (ed. Leonina). «[...] Sapere et intelligere non pertinent ad sensum, quod est ostendere sensum et intellectum non esse idem [...]». 
te a nosotros; igualmente reconocemos una sinfonía de Mozart instantáneamente, sin quizá pensar que es de Mozart.

A manera de conclusión, espero haber dado algunos argumentos para acercar a Tomás a una teoría del sentido común y matizar el intelectualismo que domina la interpretación de su epistemología. ${ }^{48} \mathrm{Se}$ vio que la teoría de los sensibles es un instrumento poderoso para determinar que los seres humanos, al igual que los demás animales, perciben el mundo sensible en toda su complejidad tal cual es. Este punto de vista realista tiene ciertas afinidades con teorías modernas, la terminología de las cuales sirvió para agudizar el pensamiento tomasiano, enmarcándolo en una discusión actual de importancia.

Jörg Alejandro Tellkemp

AA 350-226

BOGOTA- COLOMBIA

48 De la inmensa cantidad de escritos desde ese punto de vista cabe destacar un pequeño artículo; Flynn, Th., «The Cogitative Power», en The Thomist 16 (1953), pp. 542-563. 